\title{
US Pharmacopeia fighting counterfeit medicines in Africa
}

A new training centre has been set up by the United States Pharmacopeial Convention to tackle the pervasive problem of counterfeit medicines in Africa. The Center for Pharmaceutical Advancement and Training, based in Accra, Ghana, will train medicine regulatory officers, and provide expertise and develop talent so that African nations can better regulate drugs.

Reports and studies in the past three years allude to the extent of Africa's problem with counterfeit medicines. Most recently, a study assessing the quality of ergometrine and oxytocin on the Ghanaian market found that more than $94 \%$ of samples failed either assay or sterility testing (www.usp.org/sites/default /files/usp_pdf/EN/PQM/ghana -mch_mqm_report_final-mar _27_2013_rdct.pdf). Some of

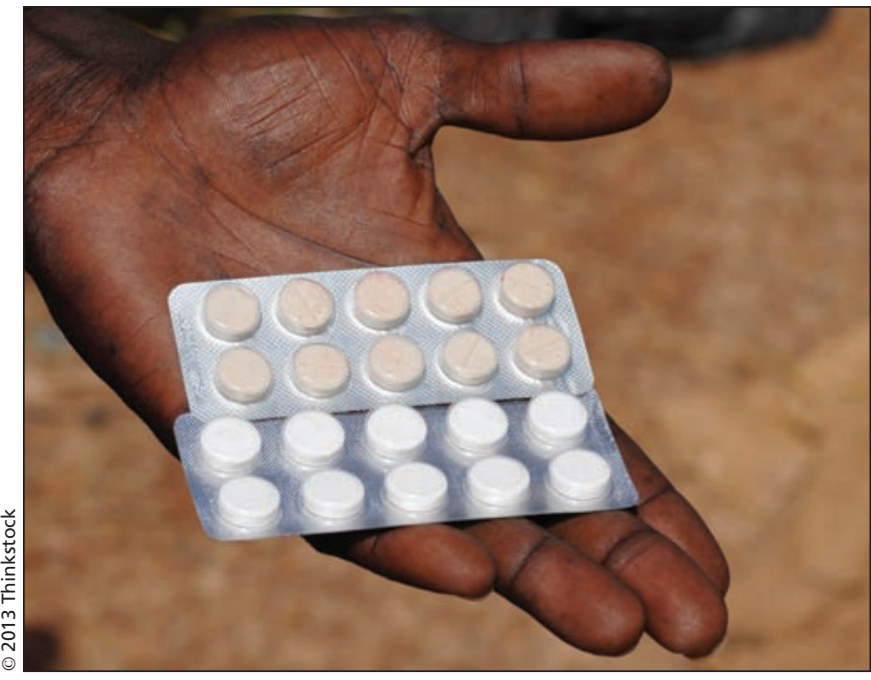

Counterfeit pharmaceuticals, particularly antimalarials, are a pervasive problem in Africa. with the right technical expertise and know-how that will make the drug regulatory agencies effective in doing the job they are supposed to do," said Dr. Patrick H. Lukulay, vice president of three years, he added. Plans for sustainability include building a quality-control laboratory to test medicines for global health development partners, local pharmaceutical companies and regulatory agencies. CePAT will charge for the testing services, and use the money to run the centre.

Samuel Kwakye, head of medicine testing laboratory at Ghana's Food and Drug Authority, said USP's ongoing collaboration with Ghana and several sub-Saharan African countries is likely to make the project attractive to African regulators. In addition, the African pharmaceutical industries' lack of adequate laboratory infrastructure for testing medicines may lead them to sign on to the project.

In Sierra Leone, Wilshire Johnson, registrar of the Pharmacy Board said that Africa needs to do more to build the capacity of drug these medicines were found
USP's Global Health Impact Programs, who also oversees CePAT operations.

To date, the project has received US $\$ 1.5$ million from USP. Six African nations' ministries of health, including those of Ghana, Ethiopia, Kenya, Senegal, Sierra Leone, and Nigeria, have signed on partly because of USP's long-term collaboration.

Initially, seven African personnel will undergo intensive training in the United States so they can train their colleagues on the continent. They will begin by offering CePAT training in dossier evaluation, good manufacturing practices and analytical techniques in laboratory work to 30 regulators; USP will foot the bill. After this initial free training, the African ministries of health will be expected to seek funding locally or from development partners to contribute to their training at the centre, said Lukulay.

CePAT will be self-sustaining after regulators to fight counterfeit medicine. "African countries should enact and enforce appropriate legislation that has strong punitive and preventive measures against counterfeiting."

There is also a need to heighten communication to help in detecting counterfeit medicines in Africa, said Dr. Stephen M. Kimatu, an official with Kenya's Pharmacy and Poisons Board. Kenya has an electronic system for reporting information on quality of medicines and adverse drug reactions for consumers and health professions and recommends that CePAT take a cue from it.

"CePAT can establish a portal where countries can actually ... share information on counterfeit medicines," says Kimatu, adding that an annual forum for sharing such information may also help African countries. - Bernard Appiah, College Station, Tex.

CMAJ 2013. DOI:10.1503/cmaj.109-4571 